\title{
Upper Extremity Arterial Thromboembolism in a Coronavirus Patient. A Case Report
}

\author{
Beverley-Ann Scott ${ }^{1}$ (D) Karan Garg ${ }^{2}$. William Johnson ${ }^{3} \cdot$ Mohammad Al-Ajam $^{4} \cdot$ Peter Patalano ${ }^{3}$. Vittorio Rotella ${ }^{4}$. \\ Jodi-Ann Edwards ${ }^{1} \cdot$ Haytham Aboushi $^{1}$ • Paul Lee ${ }^{1} \cdot$ Melissa Daniel $^{1} \cdot$ Schneider Rancy ${ }^{1} \cdot$ David Heimann $^{4}$
}

Accepted: 7 December 2020 / Published online: 7 January 2021

(C) The Author(s), under exclusive licence to Springer Nature Switzerland AG part of Springer Nature 2021

\begin{abstract}
The coronavirus disease 2019 pandemic has impacted millions of people worldwide. This novel virus has a variety of presentations and complications. Notably, patients with this infection have an associated coagulopathy, presenting with symptoms such as gastrointestinal bleeds, deep vein thrombosis, ischemic cerebrovascular events, and pulmonary embolism. Although there are documented cases of venous thromboembolism in patients with coronavirus disease 2019, the authors present an interesting case of upper extremity arterial thromboembolism in a 75-year-old patient surgically treated for arterial thrombus removal. We also discuss diagnosis, medical management, and surgical approach to an upper extremity arterial thromboembolism in a patient with coronavirus disease 2019 , to highlight the challenges of hypercoagulability in such patients.
\end{abstract}

Keywords COVID-19 coronavirus · Arterial · Thromboembolism · Coagulopathy · Hypercoagulability · Disseminated intravascular coagulopathy $\cdot$ Pulmonary embolism $\cdot$ Revascularization $\cdot$ Anticoagulation

\section{Introduction}

Since the novel coronavirus disease pandemic (COVID-19) caused by the novel coronavirus (SARS-CoV-2) began, clinicians in numerous countries have observed a wide range of

This article is part of the Topical Collection on Covid-19

Beverley-Ann Scott

Beverley-Ann.Scott@downstate.edu

Karan Garg

karan.garg@nyulangone.org

William Johnson

William.Johnson2@nyulangone.org

Mohammad Al-Ajam

Mohammad.al-ajam@va.gov

Peter Patalano

peter.patalano@nyulangone.org

Vittorio Rotella

vittorio.rotella@va.gov

Jodi-Ann Edwards

Jodi-Ann.Edwards@downstate.edu

Haytham Aboushi

Haytham.Aboushi@downstate.edu thromboembolic and hemorrhagic events [1]. Lab findings have included elevated D-Dimers and fibrinogen levels, lymphopenia, thrombocytopenia, and prolonged prothrombin times. The term CAC (COVID-associated coagulopathy) [2] has been coined to describe this phenomenon associated with

Paul Lee

paul-kyung-hyun.lee@downstate.edu

Melissa Daniel

Melissa.Daniel@Downstate.edu

Schneider Rancy

schneider.rancy@downstate.edu

David Heimann

David.Heimann@va.gov

1 SUNY Downstate Medical Center, New York, NY, USA

2 NYU Langone Hospital, New York, NY, USA

3 VA New York Harbor Health System - Manhattan, New York, NY, USA

4 VA New York Harbor Health System - Brooklyn, New York, NY, USA 
spontaneous venous thromboembolism (VTE), pulmonary embolism (PE), disseminated intravascular coagulopathy (DIC), as well as hemorrhagic events. Clinical data from early studies on patients infected with the SARS-CoV-2 in China revealed that most non-survivors had elevated D-dimer levels, suggesting a direct correlation between D-Dimer levels and prognosis.

The USA currently has the highest morbidity and mortality numbers for any country with over 11 million confirmed cases and over 250,000 COVID-19 related deaths at the time of writing [3]. Studies are examining the sudden respiratory decompensations commonly seen in these patients, which may be due to undiagnosed pulmonary embolisms [4]. What seems to be confirmed is that:

a) Coagulopathy is a marker of adverse outcome.

b) There are elements of both DIC and thrombotic microangiopathy in patients infected with the SARS-CoV-2.

c) Infection with coronavirus disease 2019 promotes a hypercoagulable state in patients which predisposes them to the venous and/or arterial thromboembolism [5].

\section{Case Presentation}

On March 31, 2020, a 75-year-old male veteran presented to NYC Health + Hospitals/Elmhurst with a low-grade fever, malaise, hypoacusis, confusion, and weakness for 2 days. His past medical history was significant for hypertension, prostate cancer, and coronary artery bypass graft. Due to the shortage of beds at NYC Health + Hospitals/Elmhurst, he was transferred to the Brooklyn Veteran's Hospital. Triage vitals were BP-156/68, P- 88, R-20. T-101.3F, SpO2 91\% on room air and improved to $96 \%$ on $2 \mathrm{~L}$ of oxygen on nasal cannula. A chest X-ray revealed patchy bilateral peripheral opacities (Fig. 1). He was admitted to the general floor for

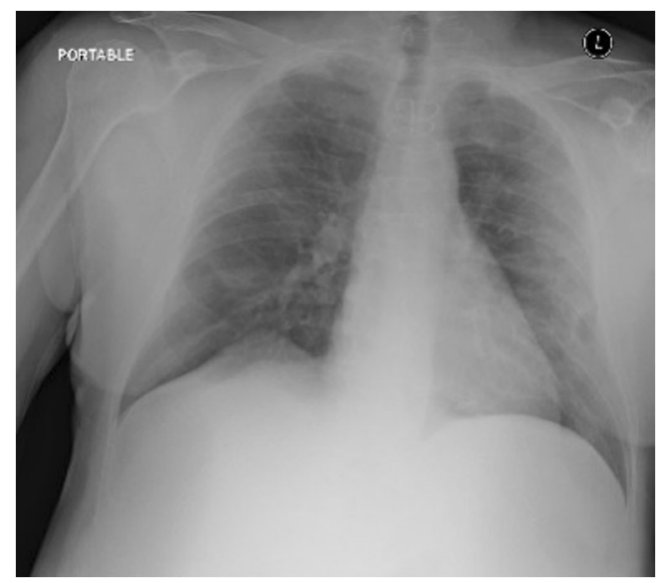

Fig 1. Unremarkable CXR March 31, 2020 suspected COVID-19 infection and received hydroxychloroquine, azithromycin, and ceftriaxone for 5 days. He was started on DVT prophylaxis of 5000 units of SC Heparin q8h. On hospital day (HD) 2, the patient remained febrile with a maximum temperature of $103 \mathrm{~F}$. A repeat chest $\mathrm{X}$-ray showed significant worsening of pulmonary infiltrates. Later that evening, he desaturated to $87 \%$ on $5 \mathrm{~L}$ of oxygen on nasal cannula, so Venturi mask at $50 \%$ was placed. He continued to show signs of respiratory distress. He was placed on Bilevel Positive Airway Pressure (BIPAP) 14/8/100\% with improvement in oxygen saturation to $96 \%$ and plans for transfer to a Medical Intensive Care Unit (MICU), with a low threshold for intubation. HD 3, he was transitioned from BIPAP to non-rebreather (NRB) mask. In the MICU, he had $\mathrm{SpO} 2$ of $93 \%$ on NRB with resolved work of breathing. Labs drawn on HD 4 were significant for AKI and transaminitis. Chest X-ray was concerning for worsening bilateral patchy infiltrates (Fig. 2.)

His AKI resolved so Heparin was discontinued, and DVT prophylaxis of Lovenox 40 units was started. The patient's mentation and inflammatory markers improved. His SARSCoV-2 resulted positive on HD 4. His transaminitis worsened and gastroenterology recommended that statin be held. On HD 7, he was started on Tocilizumab $650 \mathrm{mg}$.

On HD 9, he began to complain of pain and coldness of his right upper extremity. On exam, he had a cold and pulseless right upper extremity. A bedside Doppler ultrasound revealed a filling defect in the right brachial artery. Full heparin anticoagulation with a heparin infusion was started, and he was emergently intubated for transfer to the Manhattan VA hospital for surgical intervention. On HD 10, he underwent a right upper extremity brachial/radial and ulnar artery embolectomy; a well-formed clot was removed (Fig. 3).

In the operating room, he was found to have no collaterals to the forearm, so a cut down to the brachial bifurcation was performed. A distal brachial arteriotomy was performed, and Fogarty catheters were passed proximally and distally. The

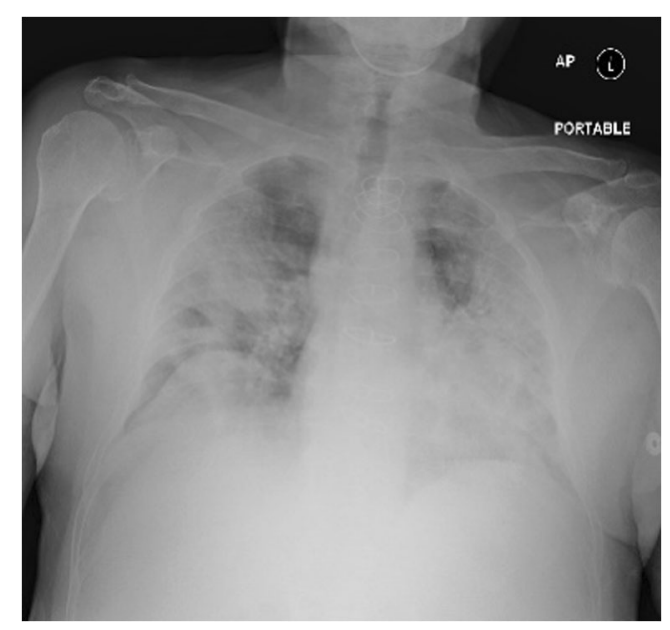

Fig. 2 CXR on April 01, 2020 


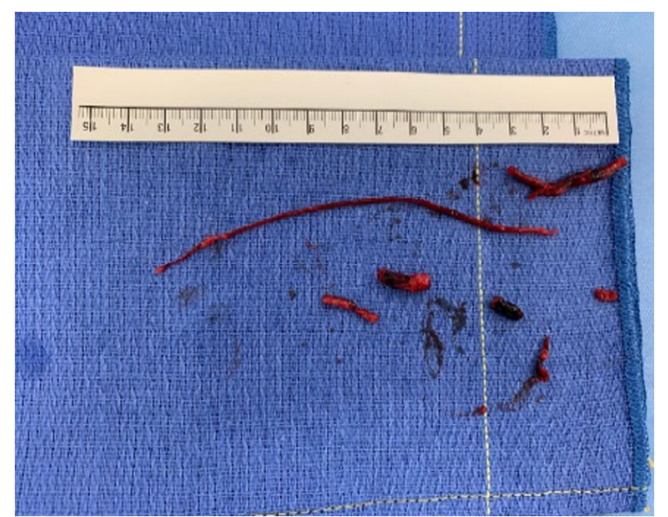

Fig. 3 Organized arterial thrombus removed from RUE on April 09, 2020

organized clots in Fig. 3 were removed and the incision was closed primarily with 6-0 Prolene. Post-operatively he was extubated but remained on high flow oxygen. Perfusion to the arm and hand was restored, and on HD 12, he was transitioned from Heparin drip to therapeutic Lovenox 80 units SC. On HD 13, he was transferred back to the Brooklyn VA. His oxygen requirements increased postoperatively. CT Thorax which was performed on his return to the Brooklyn VA showed a multilobar pneumonia, with significant increase in interstitial opacities and bronchiectasis (Fig. 4). Infectious disease was consulted, and he was started on a course of empiric Vancomycin and Cefepime for suspected nosocomial bacterial pneumonia. An echocardiogram was done that ruled out CHF. His D-dimers trended downward (Table 1), and his AKI resolved. His right upper extremity pulse exam remained unchanged with soft compartments and adequate skin perfusion; however, his oxygen requirements remained elevated. He was on high flow nasal cannula (HFNC) at 40LPM, $\mathrm{FiO} 2-50 \%$.

Table 1 D-Dimer trend. On the day of RUE Ischemia D-Dimer was significantly elevated

\begin{tabular}{ll}
\hline Date & $\begin{array}{l}\text { D- } \\
\text { Dimer }\end{array}$ \\
\hline $4 / 1 / 2020$ & 18,665 \\
$4 / 2 / 2020$ & 1222 \\
$4 / 4 / 2020$ & 800 \\
$4 / 5 / 2020$ & 1717 \\
$4 / 6 / 2020$ & 4591 \\
$4 / 8 / 2020$ & 10191 \\
$4 / 9 / 2020$ & $14,899^{*}$ \\
$4 / 12 / 2020$ & 5427 \\
$4 / 13 / 2020$ & 3327 \\
$4 / 14 / 2020$ & 2765 \\
$4 / 18 / 2020$ & 1002 \\
$4 / 21 / 2020$ & 657 \\
\hline
\end{tabular}

Although there was an interdisciplinary discussion regarding placing him on continuous positive airway pressure (CPAP), it was thought that he would not benefit from this and he was started on Dornase Alfa nebulizers x2days which improved his symptoms. HD 21, his oxygen requirement had decreased to 12LPM on 50\%HFNC. On examination of the right upper extremity, however he was noted to have an irregular, firm, non-pulsatile mass, approximately $10 \mathrm{~cm} \times$ $6 \mathrm{~cm} \times 3 \mathrm{~cm}$ in his right forearm, which represented a new area of induration, extending distally from his incision site. He also complained of new tenderness in the anterior aspect of the right forearm. His radial, ulnar, and brachial pulses remained intact although there was concern for possible hematoma.

These symptoms were accompanied by a decrease in hemoglobin from $12 \mathrm{~g} / \mathrm{dL}$ to $9.5 \mathrm{~g} / \mathrm{dL}$. On HD21, CT angiogram of the right upper extremity revealed patent right brachial, radial, and ulnar arteries without evidence of thrombus or extravasation (Fig. 5). An intramuscular and subcutaneous soft tissue hematoma in the volar and radial aspect of the proximal right forearm measuring $5.3 \times 4.0 \times 4.4 \mathrm{~cm}$ was identified in addition to soft tissue edema. The patient was advised to keep his arm elevated, and warm compresses were applied which offered relief of symptoms. CT angiogram of the chest also done on HD21 showed no evidence of pulmonary embolus and worsening of bilateral ground glass opacities with bilateral lung consolidation and trace bilateral pleural effusions. He was weaned from $50 \%$ HFNC to $50 \%$ Ventimask with oxygen saturations in the mid to low 90 s.

Around $7 \mathrm{pm}$ on HD 21, the patient reportedly had an episode of hypotension with SBP in the 60s, associated with an episode of vomiting and pain in his left thigh. A fluid bolus was administered, and the patient made NPO. At that time, his left thigh compartments were examined and found to be soft. His diet was resumed on HD 22 and he continued to be weaned off his oxygen requirements, tolerating $6 \mathrm{~L}$ on nasal cannula. Around $4 \mathrm{pm}$ on HD 22, he had two episodes of hypotension and tachycardia. The hypotension resolved with fluids, but he remained tachycardic to the 100s. His $4 \mathrm{pm} \mathrm{CBC}$ showed an elevation in his WBC to greater than 20 . Approximately 3 hours later, he had sudden onset of tachypnea, tachycardia, and hypotension, and the decision was made to transfer him to the MICU for impending respiratory failure and likely septic shock. There was also concern that he had aspirated during his episode of emesis. However, before he could be transferred to the MICU, he arrested, and CPR was started at 7:20 pm. He underwent 7 rounds of CPR and return of spontaneous circulation (ROSC) was achieved after $16 \mathrm{~min}$. He was successfully intubated and transferred to the MICU. His hemoglobin decreased to $8.3 \mathrm{~g} / \mathrm{dL}$ then 5.3, and he was transfused 2 units of packed red blood cells. CT $\mathrm{Abd} /$ Pelvis revealed no occult intra-abdominal or retroperitoneal hemorrhage. Of significance was an ill-defined $3.5 \mathrm{~cm}$ left hepatic lobe lesion. There were no new lung findings on 
Fig. 4 Coronal slices of CT Chest on April 12, 2020

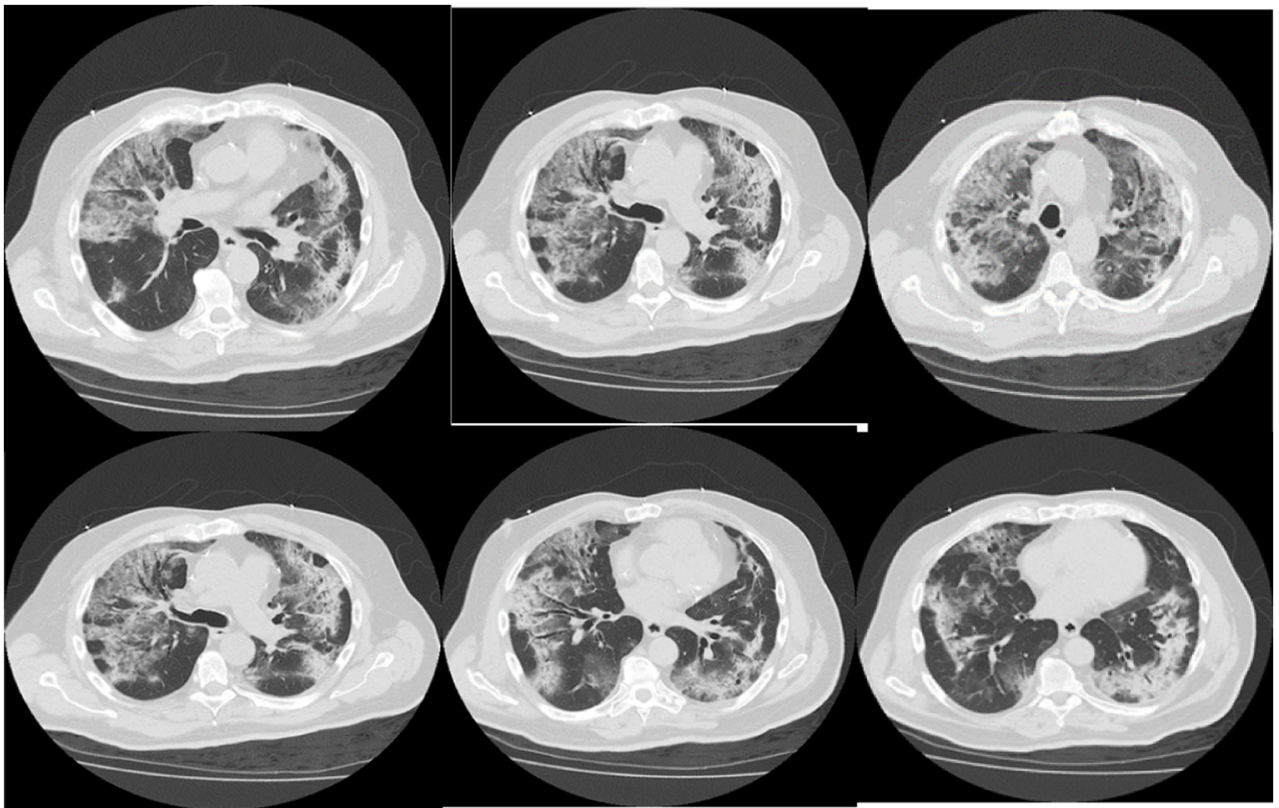

CT Chest, and he was determined to be in septic shock with likely aspiration pneumonia. After goals of care discussion with his relatives, it was agreed that he would be DNR and supportive measures only would be given. He died on April 24, 2020, HD25.

\section{Discussion}

Laboratory characteristics of patients infected with the novel coronavirus include elevations in D-Dimer levels, fibrinogen levels, and mild elevations in prothrombin time. Early studies of patients infected with the novel coronavirus in Wuhan, China, have documented poorer outcomes with the hypercoagulable state identified by some as COVID-associated coagulopathy (CAC) [6]. COVID-associated coagulopathy (CAC)

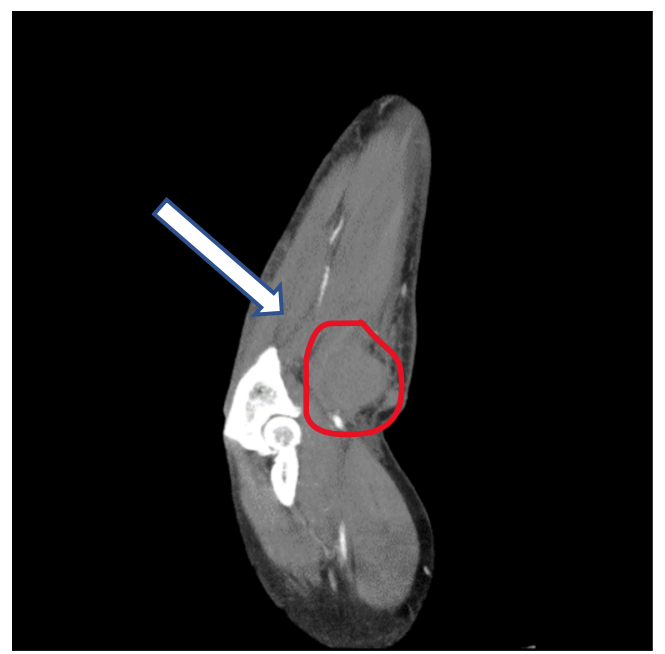

Fig. 5 CT angiogram of Right forearm hematoma on April 20, 2020 differs from DIC from other causes because the degree of elevation of the PT is much less and the thrombocytopenia is mild. Thromboembolic events such as pulmonary embolisms and deep vein thrombosis have been directly correlated to elevations in D-Dimer levels which have been a predictor of poor clinical outcome and mortality in COVID patients [4]. The cytokine storm that accompanies infection with novel coronavirus has been blamed for the increased risk of vascular hyperpermeability, multiorgan failure, and ultimately death that occurs when these pro-inflammatory cytokines are left unabated over time [7]. Early prophylactic anticoagulation with low molecular weight heparin has been recommended by the World Health Organization to prevent DVTs in COVID patients. In one retrospective study of 449 patients out of Tongji Medical College in Wuhan China, the authors found that the 28-day mortality in heparin users were lower than nonusers in patients with D-dimer $>3.0 \mu \mathrm{g} / \mathrm{mL}$ [8]. However even while on prophylactic doses of LMWH or twice daily subcutaneous unfractionated heparin, cases of pulmonary embolisms and DVTs have been reported pointing to the unique and dynamic nature of this novel coronavirus [9]. It is yet unclear whether the hemostatic changes being observed in these patients is related to the cytokine storm that triggers the systemic inflammatory response (SIRS). DVTs while on prophylactic anticoagulation are rare even in patients not infected with coronavirus. It is also unclear whether elevations in antiphospholipid antibodies play a major role in the pathophysiology of the thrombosis associated with COVID-19positive patients. While the International Society on Thrombosis and Hemostasis has recommended that all hospitalized COVID-19 patients should receive prophylactic low molecular weight heparin unless there are contraindications [10], questions still remain regarding the use of prophylactic 
doses versus full anticoagulation doses. Some studies have argued that there should be threshold levels of D-Dimer and fibrinogen levels at which anticoagulation should be started [11]. Ultimately the concern for over-anticoagulation must be balanced with the risk associated with thrombotic events in this patient population. A study by Klok et al. out of 3 Dutch hospitals which evaluated the incidence of thrombotic events in 184 ICU patients with proven COVID-19 pneumonia found that despite all patients receiving standard doses of thromboprophylaxis, the cumulative incidence of patients experiencing thrombotic complications was $31 \%$. The most common thrombotic complication was pulmonary embolism. Less common were upper and lower extremity thrombosis and ischemic strokes. [12]

\section{Conclusion}

The hypercoagulable states that have been observed in COVID-19 patients have been largely pathognomonic for venous thromboembolic events. However hemorrhagic problems may also be an initial manifestation of COVID- 19 [13]. This case report discusses an arterial thromboembolic event in a COVID-19-positive patient requiring surgical intervention. Had the event not been identified in a timely fashion, significant morbidity, and possible amputation of the extremity in question may have been the result. This case report further supports the need for early anticoagulation to prevent the sequelae of CAC and confirms that the virus is a multisystem, multi-organ disease which can predispose those infected to both venous and arterial thromboembolism. Research is still being gathered on the incidence of thrombotic complications in COVID-19 patients. The debate regarding the intensity of thromboprophylaxis required for patients admitted to the ICU who are at the highest thrombotic risk is still ongoing. While prophylactic anticoagulation has been recommended for patients with COVID-associated coagulopathy [11], it is still unclear whether standard regimens which are used for anticoagulation can be effectively applied for patients infected with the novel coronavirus. Since these patients have significantly elevated D-Dimer levels in the tens of thousands, it may be time to consider higher prophylactic doses of LMWH or UFH to empirically treat COVID-19-positive patients with full anticoagulation. The direct correlation between D-Dimer levels and poor outcomes and thromboembolic events suggests that anticoagulation protocols which correlate to these levels may be needed. Although rapid identification, evaluation, and coordination of care between the providers at the Manhattan VA and Brooklyn VA hospitals saved the upper extremity of this right-handed veteran, he eventually succumbed to multiorgan dysfunction syndrome (MODS) and overwhelming sepsis with a mixed shock picture. However, this case highlights the need to rethink the traditional understanding of prophylactic anticoagulation in these patients.

Authors' Contributions 1. Beverley-Ann Scott-primary author of manuscript, clinical care

2. Karan Garg - vascular surgery attending, clinical care and manuscript review

3. William Johnson - clinical care, manuscript review, and editing

4. Peter Patalano - clinical care and manuscript review

5. Mohammad Al-Ajam - clinical care and manuscript review

6. Vittorio Rotella — clinical care and manuscript review and editing

7. Jodi-Ann Edwards - manuscript review and editing

8. Haytham Aboushi-manuscript review

9. Paul Kyung Hyun Lee-manuscript review

10. Melissa Daniel - manuscript review

11. Rancy Schneider-manuscript review and subject matter research

12. David Heimann — clinical care and manuscript review

Data Availability Data available.

\section{Compliance with Ethical Standards}

Conflict of Interest Not applicable.

Ethical Approval "All procedures performed in studies involving human participants were in accordance with the ethical standards of the institutional and/or national research committee and with the 1964 Helsinki declaration and its later amendments or comparable ethical standards."

Consent to Participate Not applicable.

Consent for Publication Not applicable.

Code Availability Not applicable.

\section{References}

1. Joob B, Wiwanitkit V. Hemorrhagic problem among the patients with COVID-19: clinical summary of 41 Thai infected patients. Clin Appl Thromb Hemost. 2020;26:107602962091830. https:// doi.org/10.1177/1076029620918308.

2. Connors JM, Levy JH. COVID-19 and its implications for thrombosis and anticoagulation. Blood:blood.2020006000. https://doi. org/10.1182/blood.2020006000.

3. Mortality Analyses. n.d.. Retrieved April 29, 2020, from https:// coronavirus.jhu.edu/data/mortality. Accessed 26 Nov 2020

4. Léonard-Lorant I, Delabranche X, Séverac F, Helms J, Pauzet C, Collange $\mathrm{O}$, et al. Acute pulmonary embolism in COVID-19 patients on CT angiography and relationship to D-Dimer Levels. Retrieved from. 2020;296(3). https://doi.org/10.1148/radiol. 2020201561.

5. Guan WJ, Ni ZY, Hu Y, Liang WH, Ou CQ, He JX, et al. China medical treatment expert group for Covid-19. Clinical characteristics of coronavirus disease 2019 in China. N Engl J Med. 2020. https://doi.org/10.1056/NEJMoa2002032.

6. Huang C, Wang Y, Li X, Ren L, Zhao J, Hu Y, et al. Clinical features of patients infected with 2019 novel coronavirus in Wuhan,China. Lancet. 2020;395:497-506. 
7. Jose RJ, Manuel A. COVID-19 cytokine storm: the interplay between inflammation and coagulation. Lancet Respir Med. 2020;8: e46-7. https://doi.org/10.1016/s2213-2600(20)30216-2.

8. Tang N, Li D, Wang X, Sun Z. Abnormal coagulation parameters are associated with poor prognosis in patients with novel coronavirus pneumonia. J Thromb Haemost. 2020. https://doi.org/10.1111/ jth. 14768.

9. Llitjos J-F, Leclerc M, Chochois C, Monsallier J-M, Ramakers M, Auvray M, et al. High incidence of venous thromboembolic events in anticoagulated severe COVID-19 patients. 2020. https://doi.org/ $10.1111 /$ jth. 14869 .

10. Levy J, Juffermans N. Thrombotic and hemostatic issues in critical care units managing COVID-19. Retrieved from https://www.isth. org/. Accessed 27 Apr 2020.

11. Cui S, Chen S, Li X, Liu S, Wang F. Prevalence of venous thromboembolism in patients with severe novel coronavirus pneumonia. $\mathrm{J}$
Thromb Haemost Accepted Author Manuscript. 2020;18:1421-4. https://doi.org/10.1111/jth.14830.

12. Klok FA, Kruip MJHA, van der Meer NJM, et al. Incidence of thrombotic complications in critically ill ICU patients with COVID-19 [published online ahead of print, 2020 Apr 10]. Thromb Res. 2020;S0049-3848(20):30120-1. https://doi.org/10. 1016/j.thromres.2020.04.013.

13. Wada H, Thachil J, Di Nisio M, Mathew P, Kurosawa S, Gando S, et al. Guidance for diagnosis and treatment of DIC from harmonization of the recommendations from three guidelines The Scientific Standardization Committee on DIC of the International Society on Thrombosis Haemostasis. J Thromb Haemost. 2013;11:761-7. https://doi.org/10.1111/jth.12155.

Publisher's Note Springer Nature remains neutral with regard to jurisdictional claims in published maps and institutional affiliations. 